\title{
Detection of hexavalent uranium with inline and field-portable immunosensors
}

\author{
Scott J. Melton ${ }^{1}$, Haini Yu ${ }^{1}$, Mehnaaz F. Ali ${ }^{1}$, Kenneth H. Williams ${ }^{2}$, Michael J. \\ Wilkins $^{2}$, Philip E. Long ${ }^{3}$ and Diane A. Blake ${ }^{1^{*}}$ \\ ${ }^{1}$ Dept. Biochemistry, Tulane Univ. Sch. Medicine, New Orleans, LA 70112 USA \\ ${ }^{2}$ Earth Sciences, Lawrence Berkeley Laboratory, Berkeley, CA 94720 USA \\ ${ }^{3}$ Hydrology, Pacific Northwest National Laboratory, Richland, WA 99352 USA
}

\begin{abstract}
An antibody that recognizes a chelated form of hexavalent uranium was used in the development of two different immunosensors for uranium detection. Specifically, these sensors were utilized for the analysis of groundwater samples collected during a 2007 field study of in situ bioremediation in a aquifer located at Rifle, CO. The antibody-based sensors provided data comparable to that obtained using Kinetic Phosphorescence Analysis (KPA). Thus, these novel instruments and associated reagents should provide field researchers and resource managers with valuable new tools for on-site data acquisition.
\end{abstract}

\section{Introduction}

The ability to perform quantitative analyses of contaminants in groundwater samples while still in the field has been a long-term goal for environmental scientists. For uranium analysis, samples must be transported off-site for any complex, detailed analysis such as ICP-MS or AAS. Simpler instrumentation like the Kinetic Phosphorescence Analyzer (KPA) is also used primarily in a laboratory setting; in addition, this instrument is useful only for the analysis of uranium and lanthanides (Brina and Miller, 1993). Here we describe two immunosensors that can be adapted for uranium analysis through the use of antibodies that bind to a $\mathrm{UO}_{2}{ }^{2+}$ chelate complex.

Immunoassays have numerous advantages for quantifying levels of environmental contaminants. Immunoassay methods are rapid and simple to perform. Relatively compact instruments can be designed to quantify antibody binding; such instruments are thus amenable for use in a field setting. Finally, the immunosensors used in the experiments described herein can be modularized such that many different contaminants can be measured using an identical sensor plat- 
2 Scott J. Melton1, Haini Yu1, Mehnaaz F. Ali1, Kenneth H. Williams2, Michael J.

Wilkins2, Philip E. Long3 and Diane A. Blake1*

form; if an antibody to a specific environmental contaminant can be generated, it can be used with this sensor technology. In the present study, two instruments based on the principle of kinetic exclusion (Blake 1999, Kusterbeck and Blake 2008) were used to assay groundwater samples from a uranium-contaminated site in Rifle, CO. Both immunosensors were able to detect changes in uranium levels during an in situ remediation process and thus show promise towards eventual field deployment for a variety of environmental sensing needs.

\section{Methods}

\section{Materials}

The uranium-selective chelator 2,9-dicarboxyl-1,10-phenanthroline (DCP) was purchased from Alfa Aesar (Ward Hill, MA). 12F6, a mouse monoclonal antibody that binds specifically a $\mathrm{UO}_{2}{ }^{2+}$-DCP complex, and an immobilized form of chelated uranium $\left(\mathrm{UO}_{2}{ }^{2+}\right.$-DCP-BSA conjugate) were available from a previous study (Blake et al. 2004). A Cy5-labeled Fab of goat anti-mouse IgG was obtained from Jackson ImmunoResearch Laboratories (Gaithersburg, MD). Bisacrylamide/azlactone copolymer beads (UltraLink Biosupport), used in the Inline sensor, were a product of Pierce Biotechnology (Rockford, IL). Polystyrene beads, used with the field portable device (FPD), were acquired from Sapidyne Instruments, Inc (Boise, ID). The diameter of both bead types was $\sim 98 \mu \mathrm{m}$; these beads were coated with the $\mathrm{UO}_{2}{ }^{2+}$-DCP-BSA conjugate by procedures that have been previously described (Blake et al, 2004; Yu et al, 2005). $\mathrm{A} \mathrm{UO}_{2}{ }^{2+}$ standard was made from uranyl acetate obtained from Mallinckrodt (St. Louis, MO). Environmental water samples were obtained in August and September, 2007 from a sampling well (D-02) at the Rifle UMTRA site, Rifle CO. The collected samples $(\sim 50 \mathrm{ml}$ each) were filtered through a $0.2 \mu \mathrm{M}$ IC MILLEX-LG syringe filter (Millipore, Billerica, MA) and refrigerated. All samples were acidified with $8 \mathrm{~N} \mathrm{HNO}_{3}$ to a $\mathrm{pH}$ of 2 before analysis. Standard curves were generated using a 1:200 dilution of "Rifle Artificial Ground Water" (RAGW), made from a formulation developed by K.M. Campbell of the U.S. Geological Survey (Menlo Park, CA).

\section{Inline Sensor}

The Inline sensor, developed in conjunction with Sapidyne Instruments (Boise, ID) (Fig. 1A) is an instrument designed to be operated in a process line capacity (Yu et al., 2005; Bromage et al., 2007; Kusterbeck and Blake, 2008). 

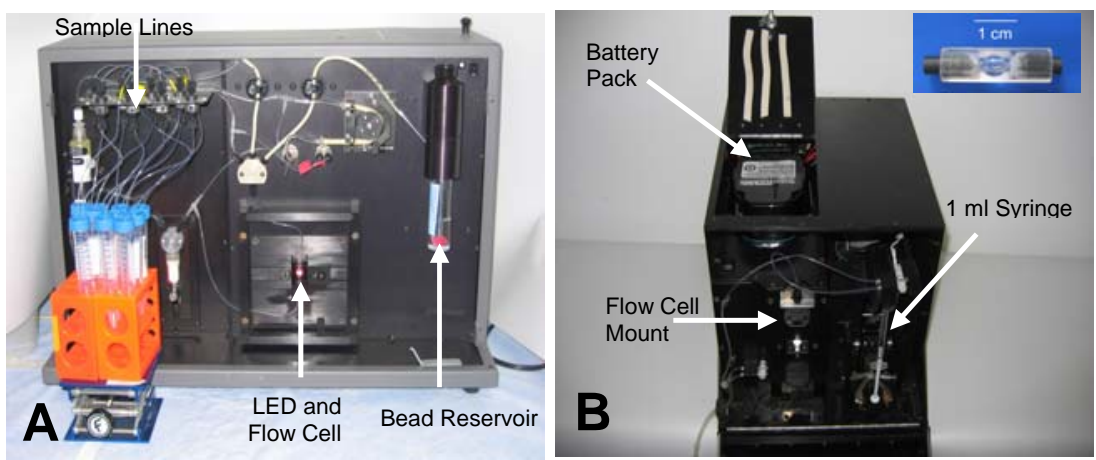

Fig 1 Uranium immunosensors. A, The KinExA Inline sensor (footprint, 30x56 cm) autonomously mixes assay components and injects them over a capillary bead column illuminated by an LED. The instrument measures fluorescently labeled antibody bound to the column; multiple samples can be assayed in one experimental run. B, The Field Portable Device (footprint, $23.5 \times 32 \mathrm{~cm}$ ) is a self-contained instrument that injects operatorprepared samples from a loaded $1 \mathrm{ml}$ syringe over a pre-filled flow cell (inset). The instrument with battery weighs approximately 6 kilograms; a carrying case with room for all necessary accessories (not shown) increases portability.

The Inline sensor required a grounded power source and was able to autonomously mix all components, run a standard curve, and analyze unknowns. Bisacrylamide/azlactone copolymer beads $(50 \mathrm{mg})$ were coated with $\mathrm{UO}_{2}{ }^{2+}$-DCPBSA conjugate and loaded into the bead reservoir before the assay sequence was initiated. $\mathrm{UO}_{2}{ }^{2+}$ was spiked into Hepes-buffered saline (HBS, $137 \mathrm{mM} \mathrm{NaCl}, 3$ $\mathrm{mM} \mathrm{KCl}, 10 \mathrm{mM}$ Hepes, $\mathrm{pH}$ 7.4) containing $200 \mathrm{nM}$ DCP and a 1:200 dilution of RAGW in order to generate the standard curve. Environmental samples were diluted 1:200 in Hepes-buffered saline containing $200 \mathrm{nM}$ DCP. The $\mathrm{pH}$ of the environmental samples after a 1:200 dilution into HBS was between 7.0 and 7.2. All assay mixtures also contained the anti-uranium antibody $12 \mathrm{~F} 6(0.25 \mathrm{nM})$ and Cy5Fab ( $5 \mathrm{nM}$, used to fluorescently label 12F6). The signals generated by the environmental samples were compared to the standard curve to determine concentrations of $\mathrm{UO}_{2}{ }^{2+}$. The instrument was programmed ( $\mathrm{Yu}$ et al, 2005) to generate a five-point standard curve and analyze seven samples in a single experimental run. All data points (standards and environmental samples) were obtained in triplicate.

\section{Field Portable Device}

The field portable device (FPD) (Fig. 1B), also developed in conjunction with Sapidyne Instruments (Kusterbeck and Blake, 2008), was designed to be used in the field without the need for a grounded power supply. Instead, the device was powered with a power drill battery available at most hardware stores. The instru- 
4 Scott J. Melton1, Haini Yu1, Mehnaaz F. Ali1, Kenneth H. Williams2, Michael J.

Wilkins2, Philip E. Long3 and Diane A. Blake1*

ment was completely enclosed in a plastic case and controlled by laptop through a wireless interface. Unlike the re-usable capillary flow cell utilized by other kinetic exclusion instruments (Blake et al., 2004; Yu et al., 2005), the FPD used a disposable flow cell (Fig. 1B, inset) prefilled with polystyrene beads coated with the $\mathrm{UO}_{2}{ }^{2+}$-DCP-BSA conjugate. Assay components were mixed by the operator; the final concentrations of the reagents were as described for Inline sensor analysis, except the Cy5-Fab concentration was reduced to $2.5 \mathrm{nM}$. Due to the limited binding capacity of the flow cell, the data points of the standard curve were obtained in singlet, while the environmental sample was analyzed in triplicate.

\section{Kinetic Phosphorescence Analysis}

In order to validate the performance of the immunosensors described herein, acidified groundwater samples were also analyzed with a kinetic phosphorescence analyzer (KPA) and Uraplex reagent available from ChemCheck Instruments (Bellingham, WA). Each sample was measured at three dilutions to ensure accuracy.

\section{Results}

The Inline sensor and the FPD are both flow fluorimeters that employ the kinetic exclusion method. This method measures the concentration of free, uncomplexed antibody in assay mixtures containing fluorescently-labeled antibody, the contaminant of interest, and antibody-contaminant complexes (Blake et al, 1999). A structural analogue of the contaminant (in this case chelated uranium) was coated onto beads. These beads were subsequently packed into a flow cell and used to capture the free fluorescently-labeled antibody; the fluorescence on the beads was monitored as the assay mixture flowed through the cell.

Typical data traces for the FDP are shown in Fig 2. This instrument recorded the baseline fluorescence 5 seconds prior to injection of the sample. Sample injection was completed in $\sim 50$ seconds and the instrument then automatically rinsed the flow cell from a buffer reservoir. The instrument automatically determined the baseline signal from the first 5 seconds of the trace and subtracted that value from the final signal after the rinse to generate a "delta" signal, which was inversely proportional to the amount of $\mathrm{UO}_{2}{ }^{2+}$ in the sample. A delta signal for each sample could be determined in 140 seconds.

In order to generate a standard curve, known amounts of $\mathrm{UO}_{2}{ }^{2+}$ were added to a buffered sample that contained $200 \mathrm{nM}$ DCP chelator and RAGW in the same dilution as that used for the environmental samples. The chelated $\mathrm{UO}_{2}{ }^{2+}$ bound to fluorescently labeled 12F6 antibodies present in the sample; these bound antibodies were therefore not available for binding to the chelated $\mathrm{UO}_{2}{ }^{2+}$ immobilized on the beads. As more soluble $\mathrm{UO}_{2}{ }^{2+}$ was added to the assays, less fluorescently labeled antibody was bound to the beads. This competition for limited antibody 
binding sites resulted in a delta signal from the instrument that was inversely proportional to the amount of $\mathrm{UO}_{2}{ }^{2+}$ present in the sample.

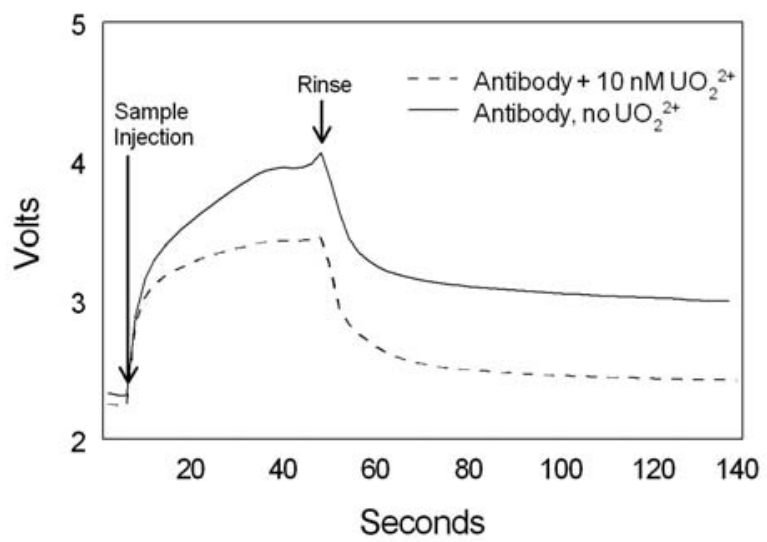

Fig 2. Primary data traces from the FPD. Solid line, sample containing antibody but no $\mathrm{UO}_{2}{ }^{2+}$. Dashed line, sample containing antibody plus a $\mathrm{UO}_{2}{ }^{2+}$ concentration high enough to fill all antibody binding sites $(10 \mathrm{nM})$.

A 4-point standard curve was generated using the FPD, as shown in Fig 3.

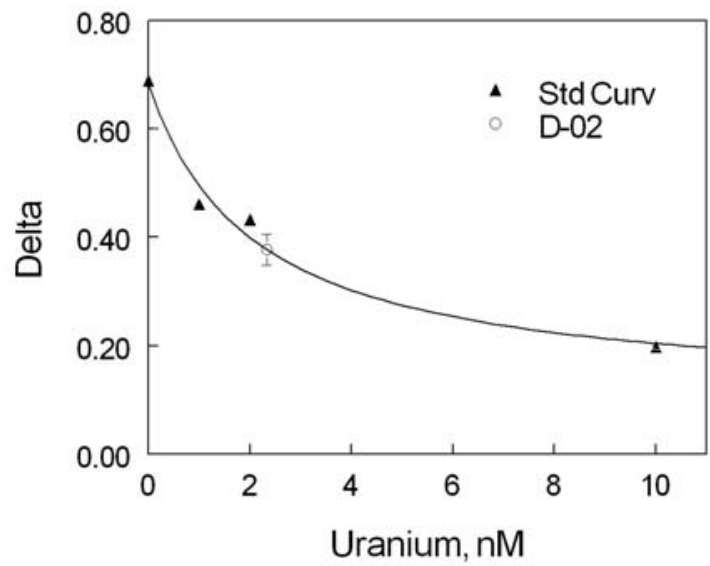

Fig 3. Uranium standard curve developed using the FPD. $\mathrm{UO}_{2}{ }^{2+}$ standards (closed triangles) and a sample from a sampling well in Rifle (well D-02, open circle) were prepared as described in Methods. The concentration of $\mathrm{UO}_{2}{ }^{2+}$ present in the sample after factoring in the dilution was $473.1+/-87.9 \mathrm{nM}$. Points obtained for generation of the standard curve were singlets while the environmental sample was run in triplicate. 
6 Scott J. Melton1, Haini Yu1, Mehnaaz F. Ali1, Kenneth H. Williams2, Michael J.

Wilkins2, Philip E. Long3 and Diane A. Blake1*

The standard curves were fit to the data points using the following equation:

$$
Y_{A}=\frac{a 0-(a 1 * x)}{a 2+x}
$$

in which $\mathrm{Y}_{\mathrm{A}}$ is the delta value at a particular concentration of $\mathrm{UO}_{2}{ }^{2+}, \mathrm{a} 0$ is the delta at an infinite concentration, a1 is the magnitude of change in delta from the lowest to the highest $\mathrm{UO}_{2}{ }^{2+}$ concentrations and a2 is the concentration of $\mathrm{UO}_{2}{ }^{2+}$ that results in $50 \%$ inhibition of the signal. The a 2 is also the equilibrium dissociation constant $\left(\mathrm{K}_{\mathrm{d}}\right)$ of $12 \mathrm{~F} 6$ binding to the $\mathrm{UO}_{2}{ }^{2+}-\mathrm{DCP}$ complex. Since this value has been determined in a previous study (Blake et al., 2004), the a2 obtained was a reliable indicator of the accuracy of the standard curve. The amount of $\mathrm{UO}_{2}{ }^{2+}$ in an environmental sample was determined by diluting the environmental sample into HBS buffer containing the same reagents used for the standard curve. The sample was then injected over the beads and the resultant data point was fitted onto the standard curve.

Similar analyses were also performed using the Inline sensor. In contrast to the FPD, which used a single bead pack in a disposable flow cell for multiple measurements, the Inline sensor used a fresh set of beads for each measurement. Beads were stored as a slurry in a reservoir bottle (shown in Fig 1A) and the instrument automatically packed a new bead microcolumn at the beginning of each measurement. Typical data traces from the Inline sensor are shown in Fig. 4. The inset shows a uranium standard curve prepared by plotting delta versus uranium concentration.

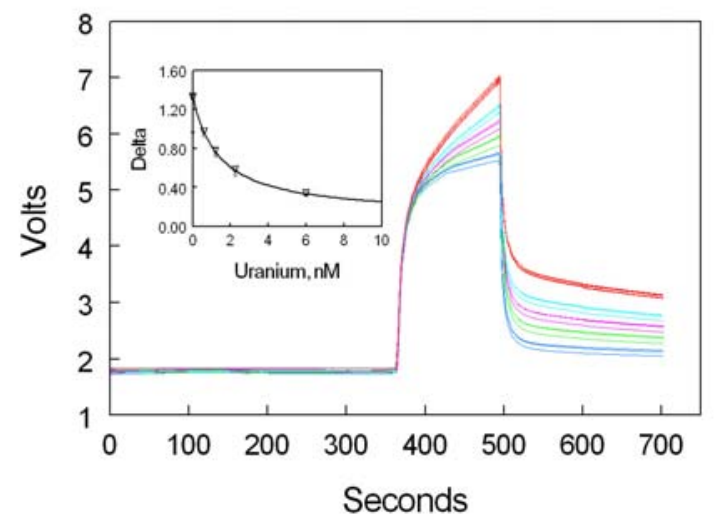

Fig 4. Data traces and uranium standard curve from the Inline sensor. The instrument packed beads into the flow cell, washed sample lines and tubes and mixed experimental samples from stock solutions from 0-359 sec. Sample injection occurred at 360 seconds and was followed by a buffer rinse. Inset, Uranium standard curve. All experiments were performed in triplicate. 
Finally, both the FPD and the Inline sensor were compared with kinetic phosphorescence analysis for their ability to assess uranium in environmental groundwater samples. These samples were obtained during an in situ bioremediation experiment conducted at the Uranium Mill Tailings Remedial Action (UMTRA) site located in Rifle, CO. Detailed descriptions of the history, geology and hydrogeology of this site have been described elsewhere (Anderson, et al., 2003; Vrionis, et al., 2005). Background groundwater concentrations of uranium are approximately 500 to $1000 \mathrm{nM}$. A series of monitoring wells were installed down-gradient of an injection gallery. This injection gallery, installed perpendicular to the groundwater flow, was used to pump acetate into the aquifer. Biostimulation with acetate is thought to initially stimulate the growth of Geobacter species, which are able to reduce soluble $\mathrm{U}(\mathrm{VI})$ to insoluble $\mathrm{U}(\mathrm{IV})$ and decrease the uranium in the water column (N'Guessan et al., 2008). Groundwater samples were collected at intervals after initiation of acetate injection and analyzed for uranium. Fig 5 shows the levels of soluble uranium in a representative downstream well, D-02, during continuous acetate injection from the day 0 to day 35. As reported previously (N'Guessan, 2008) acetate injection caused a relatively sharp decrease in soluble uranium that was detected with all three instruments. Additionally, the data from the two immunosensors correlated well with the data from the KPA.

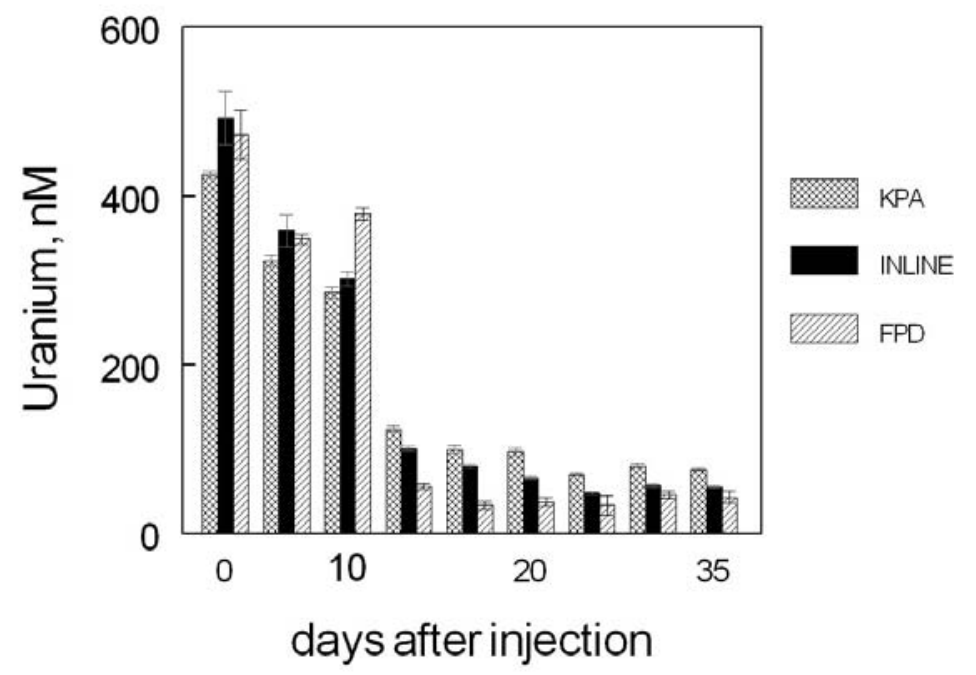

Fig 5. Comparison of KPA, Inline, and FPD analysis. Groundwater samples were collected at the indicated times after the initiation of acetate injection, filtered and acidified. KPA analyses were performed at 3 dilutions; samples were analyzed in triplicate using the Inline Sensor and FPD. The error bars represent the standard error of the mean. 
8 Scott J. Melton1, Haini Yu1, Mehnaaz F. Ali1, Kenneth H. Williams2, Michael J.

Wilkins2, Philip E. Long3 and Diane A. Blake1*

\section{Discussion}

Previous work in our laboratory has focused on the isolation/characterization of antibodies that bind to metal-chelate complexes and on the development of antibody-based assays useful for measuring a variety of heavy metals in a given sample (Khosraviani et al., 1998; Delehanty et al., 2003; Darwish and Blake, 2004; Kriegel et al., 2006; Zhu et al., 2007). The work described in this report represents some of the first experiments carried out by our laboratory in a field setting and demonstrates the portability, speed and overall utility of immunosensors for environmental analysis. However, as with all immunoassays, a detailed understanding of the binding properties of the antibody used in the assay is vital for the success of this method.

Monoclonal antibodies that recognize environmental contaminants are typically generated in mice by repeated exposure of the contaminant (or a structural analogue of the contaminant) to the mouse immune system. Metal cations are too small to illicit an immune response and our laboratory has developed a method whereby an immunogen is prepared by immobilizing the metal via a bifunctional chelator to a carrier protein (for a review, see Blake et al., 2007). The antibodies generated from such immunizations recognize metals bound to a chelator, rather than free metals. Since metals in environmental samples almost always exist in a complexed state, an important part of any assay development effort is devising a strategy that removes the metal from its natural complexants and transforms it to a form recognized by the antibody. The antibody used for the uranium analysis, $12 \mathrm{~F} 6$, recognizes uranium in a complex with DCP (Blake et al, 2004). Thus, the uranium in the environmental samples from the Rifle site had to be dissociated from complexants present in the groundwater samples and subsequently transformed to DCP complexes. While the optimal pre-treatment strategy for Rifle samples (acidification, then neutralization into buffers containing DCP) was not determined until after the field experiment had been completed, future experiments should allow for near real-time quantification of uranium in the field.

The use of the Inline sensor has both advantages and disadvantages for field use. This instrument had a relatively high sample throughput and provided data with minimal effort on the part of the operator. In a typical day at Rifle, we collected and pretreated samples during a day of field work; the Inline sensor was then programmed to analyze them overnight. The instrument's autonomous operation and relatively small footprint $(30 \times 56 \mathrm{~cm})$ was advantageous in the cramped conditions that existed in our field laboratory (a converted horse trailer). The Inline sensor provided data that was as precise as larger immunoassay instruments in our laboratory. This superior precision could be attributed to the instrument's ability to prepare a fresh set of reagents (bead column, freshly mixed assay components) for each measurement; however, use of fresh reagents limited the total number of individual samples per run to $\sim 50$. The main disadvantage of the Inline sensor was its requirement for a grounded $110 \mathrm{AC}$ power source.

Because of its independent power supply and wireless interface, the FPD could be operated in the absence of a grounded power supply (although the drill 
batteries used for operation needed to be recharged either from an automotive battery or a grounded power source). The instrument is comparatively light (6 kg); it was transported in a backpack-like bag that also had room for all necessary reagents and accessories (pipettes, syringes, disposable tubes); thus, this instrument could be used in a remote setting. One of the issues currently being addressed during further FPD development is the binding capacity of the disposable flow cells supplied with the instrument. Unlike the Inline immunosensor, the FPD uses the same bead column for multiple measurements. This ultimately results in a decrease in instrument responsiveness as more and more antibody binds to the $\mathrm{UO}_{2}{ }^{2+}$-DCP coated on the beads. For the experiments described herein, we responded by limiting the number of standards and experimental samples analyzed on each disposable flow cell. In practical terms, these limitations decreased precision due to fewer replicate measurements. A fresh flow cell was required for every environmental sample, which led to a decrease in sample throughput. New bead coating strategies and sample injection schemes are being explored to optimize instrument performance.

The data obtained with these two new immunosensors compared well that obtained using KPA. As seen in Fig 5, the data from both the Inline and FPD correlated well with the KPA analysis of the D-02 test well, especially at the higher uranium levels seen at the beginning of this study. While the FPD, in particular, was less able to monitor uranium at lower levels, both the Inline sensor and the FPD were able detect the removal of uranium from the groundwater sample collected during experiments performed during the summer of 2007.

The immunosensors described herein can be easily adapted to the analysis of a wide variety of other experimental contaminants. Assays for other heavy metals, PCB's, 2,4-dichlorophenoxyacetic acid, environmental estrogens, organophosphate pesticides, imidazolinone herbicides and TNT have been published using the $\mathrm{KinExA}^{\mathrm{TM}}$ technology employed by the Inline sensor and FPD (for a review, see Kusterbeck and Blake, 2008). These new field deployable sensors will provide researchers and resource managers with an invaluable tool for generating near realtime data and modifying field experiments already in progress.

\section{Acknowledgments}

This work was supported by the Office of Science (BER) of the U.S. Department of Energy, Grant DE-FG98-ER62704 (D.A.B.), and DE-AC02-05CH11231. The field experiment it was based on was conducted as part of the U.S. DOE's Integrated Field Challenge Site (IFC) at Rifle, Colorado. The Rifle IFC is a multidisciplinary, multi-institutional project supported by the Environmental Remediation Sciences Division of Biological and Environmental Research, Office of Science, U.S. Department of Energy. The project is lead by Pacific Northwest National Laboratory and includes a number of university and national laboratory partners. The authors thank Kate M. Campbell of the USGS, Menlo Park, CA, for providing the Rifle artificial groundwater formulation. 
10 Scott J. Melton1, Haini Yu1, Mehnaaz F. Ali1, Kenneth H. Williams2, Michael J. Wilkins2, Philip E. Long3 and Diane A. Blake1*

\section{References}

Anderson RT, Vrionis HA, Ortiz-Bernad I, Resch CT, Long PE, Dayvault R, Karp K, Marutzky S, Metzler DR, Peacock A, White DC, Lowe M, Lovley DR (2003) Stimulating in situ activity of Geobacter species to remove uranium from the groundwater of a uranium-contaminated aquifer. Appl Environ Microbiol 69: 5884-5891

Blake, DA, Blake, RC $2^{\text {nd }}$, Abboud ER, Li X, Yu H, Kriegel AM, Khosraviani M, Darwish IA (2007) "Antibodies to heavy metals: Isolation, characterization and incorporation into microplate-based assays and immunosensors". In Immunoassay and Other Bioanalytical Techniques (Ed. J.M. Van Emon), Taylor and Francis, Boca Raton, FL, pp. 93-111

Blake RC $2^{\text {nd }}$, Pavlov AR, Blake DA (1999) Automated kinetic exclusion assays to quantify protein binding interactions in homogeneous solution. Anal Biochem 272: 123-134

Blake RC $2^{\text {nd }}$, Pavlov AR, Khosraviani M, Ensley HE, Kiefer GE, Yu H, Li X, Blake DA (2004) Novel monoclonal antibodies with specificity for chelated uranium(VI): Isolation and binding properties. Bioconj Chem 15: 1125-1136

Brina R, Miller AG (1993) Determination of uranium and lanthanides in real-world samples by kinetic phosphorescence analysis. Spectroscopy 8: 25-28

Bromage ES, Lackie T, Unger MA, Ye J, Kaattari SL (2007) The development of a realtime biosensor for the detection of trace levels of trinitrotoluene (TNT) in aquatic environments. Biosensor Bioelectron 22: 232-238.

Darwish IA, Blake DA (2002) Development and validation of a sensitive one-step immunoassay for determination of cadmium in human serum. Anal Chem 74: 52-58

Delehanty JB, Jones RM, Bishop TC, Blake DA. (2003) Identification of important residues in metal-chelate recognition by monoclonal antibodies. Biochemistry 42: 1417314183

Khosraviani M, Pavlov AR, Flowers GC, Blake DA (1998) Detection of heavy metals by immunoassay: Optimization and validation of a rapid, portable assay for ionic cadmium. Env Sci Technol 32: 137-142

Kriegel AM, Soliman AS, Zhang Q, El-Ghawalby N, Ezzat F, Soultan A, Abdel-Wahab M, Fathy O, Ebidi G, Bassiouni N, Hamilton SR, Abbruzzese JL, Lacey MR, Blake DA. (2006) Serum cadmium levels in pancreatic cancer patients from the East Nile Delta region of Egypt. Environ Health Perspect 114: 113-119

Kusterbeck AW, Blake DA (2008) "Flow immunosensors" in Optical Biosensors: Today and Tomorrow (Ed, Ligler FS, Tait CR ), Elsevier, New York, pp 243-285

N'Guessan AL, Vrionis HA, Resch CT, Long PE, Lovley DR (2008) Sustained removal of uranium from contaminated groundwater following stimulation of dissimilatory metal reduction. Env Sci Tech 42: 2999-3004

Vrionis HA, Anderson RT, Ortiz-Bernad I, O’Neill KR, Resch CT, Peacock AD, Dayvault R, White DC, Long PE, Lovley DR (2005) Microbiological and geochemical heterogeneity in an in situ bioremediation field site. Appl Environ Microbiol 71: 6308-6318

Yu H, Jones RM, Blake DA (2005) An immunosensor for autonomous in-line detection of heavy metals: Validation for hexavalent uranium. Int J Env Anal Chem 85: 817-830

Zhu X, Hu B, Lou Y, Xu L, Yang F, Yu H, Blake DA, Liu F (2007) Characterization of monoclonal antibodies for lead-chelate complexes: applications in antibody-based assays. J Agric Food Chem 55: 4993-4998 